\title{
Psoriasiform dermatitis is driven by IL-36-mediated DC-keratinocyte crosstalk
}

\author{
Luigi Tortola, ${ }^{1}$ Esther Rosenwald,, ${ }^{1}$ Brian Abel, ${ }^{1}$ Hal Blumberg, ${ }^{2}$ Matthias Schäfer, ${ }^{3}$ \\ Anthony J. Coyle, ${ }^{4}$ Jean-Christoph Renauld, ${ }^{5}$ Sabine Werner, ${ }^{3}$ Jan Kisielow, ${ }^{1}$ and Manfred Kopf ${ }^{1}$ \\ 1Institute of Molecular Health Sciences, Molecular Biomedicine, Swiss Federal Institute of Technology (ETH) Zürich, Zürich, Switzerland. \\ ${ }^{2}$ Novo Nordisk Inflammation Research Center, Seattle, Washington, USA. IInstitute of Molecular Health Sciences, Cell Biology, ETH Zürich, Zürich, Switzerland. \\ ${ }^{4}$ Pfizer Inc., Cambridge, Massachusetts, USA. ${ }^{5}$ Ludwig Institute for Cancer Research, Brussels Branch, Brussels, Belgium.
}

\begin{abstract}
Psoriasis is a chronic inflammatory disorder of the skin affecting approximately $2 \%$ of the world's population. Accumulating evidence has revealed that the IL-23/IL-17/IL-22 pathway is key for development of skin immunopathology. However, the role of keratinocytes and their crosstalk with immune cells at the onset of disease remains poorly understood. Here, we show that IL-36R-deficient $\left(\mathrm{Il} 36 \mathrm{r}^{-/-}\right)$mice were protected from imiquimod-induced expansion of dermal IL-17-producing $\gamma \delta T$ cells and psoriasiform dermatitis. Furthermore, IL-36R antagonist-deficient (Il36rn-/-) mice showed exacerbated pathology. TLR7 ligation on DCs induced IL-36-mediated crosstalk with keratinocytes and dermal mesenchymal cells that was crucial for control of the pathological IL-23/IL-17/IL-22 axis and disease development. Notably, mice lacking IL-23, IL-17, or IL-22

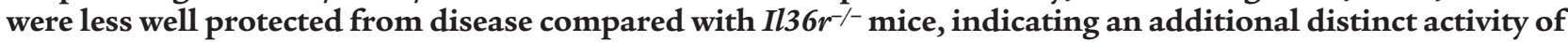
IL-36 beyond induction of the pathological IL-23 axis. Moreover, while the absence of IL-1R1 prevented neutrophil infiltration, it did not protect from acanthosis and hyperkeratosis, demonstrating that neutrophils are dispensable for disease manifestation. These results highlight a central and unique IL-1-independent role for IL-36 in control of the IL-23/IL-17/IL-22 pathway and development of psoriasiform dermatitis.
\end{abstract}

\section{Introduction}

Psoriasis is the most common chronic inflammatory skin disease, which is characterized by epidermal hyperplasia (acanthosis) due to hyperproliferation and impaired differentiation of keratinocytes, scaling, and erythematous plaque formation, eventually resulting in loss of the protective skin barrier $(1,2)$. Another defining histological feature is an inflammatory infiltrate, consisting of neutrophils and mononuclear cells in the dermis and epidermis. However, little is known about the molecular mechanisms controlling the initial stages of psoriasis. According to the current understanding, a cross talk between skin-resident DCs and T cells, infiltrating macrophages and neutrophils, and keratinocytes through inflammatory mediators is thought to be responsible for the aberrant keratinocyte proliferation and differentiation and disease development. The precipitating event is the activation of DCs through TLR ligation by microbes or other environmental factors and the release of IL-12 and IL-23 that drive polarization into effector T cells secreting TNF- $\alpha$, IFN- $\gamma$, IL-17, and/or IL-22. These cytokines cooperatively stimulate keratinocytes to produce a variety of growth factors and inflammatory mediators, ultimately fuelling the vicious cycle of cutaneous pathology (3).

Despite the inherent differences between human and mouse skin, substantial insights into the underlying mechanisms of skin inflammation have been gained using xenotransplantation of human psoriatic skin to immunodeficient mice $(4,5)$ or several mouse models of psoriasis-like disorders. The latter include $\mathrm{T}$ cell transfer into CB17 SCID/SCID mice (6); transgenic overexpression

Authorship note: Jan Kisielow and Manfred Kopf contributed equally to this work. Conflict of interest: Hal Blumberg and Anthony J. Coyle are employees of Novo Nordisk and Pfizer, respectively.

Citation for this article: J Clin Invest. 2012;122(11):3965-3976. doi:10.1172/JCI63451. of several cytokines, such as IL-12p40 (7), IL-1 $\alpha$ (8), and TGF- $\beta$ (9), specifically in keratinocytes; dermal injection of IL-23 (10); and treatment with the TLR7 agonist imiquimod (IMQ) (11), which is clinically relevant as it is applied topically as a drug against skin cancer and known to induce psoriasis in susceptible patients $(12,13)$. Together, these models revealed that the cytokines IL-23, IL-17, and IL-22 (also termed the IL-23/IL-17/IL-22 axis) are key mediators of psoriasiform disease $(6,7,10,11,14-16)$, with IL-23 secreted by some sort of DC which is responsible for development of pathological IL-17A- and IL-22-producing T cells. Notably, besides Th17 cells, IL-17 and IL-22 can be produced by $\gamma \delta \mathrm{T}$ cells $(17,18)$ and NKT cells $(19)$, which all require IL-1 together with IL-23 for expansion (20-22). An important role of IL-23 and IL-17 in human psoriasis has become evident from genome-wide studies and the efficacy of biologics targeting these cytokines (23-26). Despite the well-accepted relevance of the IL-23/IL-17/IL-22 axis, no study has directly compared the individual knockout mice side by side in a psoriasis model to understand their relative contribution and relevance for disease development.

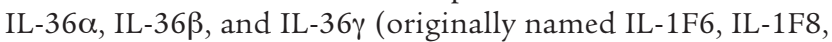
and IL-1F9, respectively) are novel members of the IL-1 family of cytokines. Each binds specifically to the IL-36 receptor (IL-36R; also named IL-1Rrp2 or IL-1RL2), which leads to the recruitment of IL-1RAcP and activation of NF- $\mathrm{KB}$. An IL-36 receptor antagonist (IL-36RN), also termed IL-1F5, inhibits signaling by binding to IL-36R $(27,28)$. Accumulating evidence implicates IL-36 cytokines in the development of psoriasis. Increased levels of IL-36 cytokines and IL-36R were observed in lesions of human psoriatic skin and confirmed in mouse models of psoriasis-like diseases (28-30). IL-36 $\alpha$ overexpression in keratinocytes of K14-IL-36 $\alpha$ transgenic mice was reported to result in a transient inflammatory skin disorder at birth that waned after 2 to 3 weeks of age (31) but rendered mice highly susceptible to 12 -O-tetrade- 
A

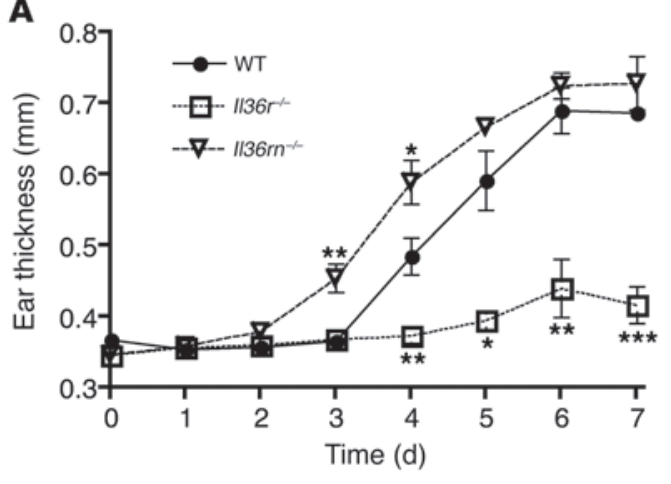

B
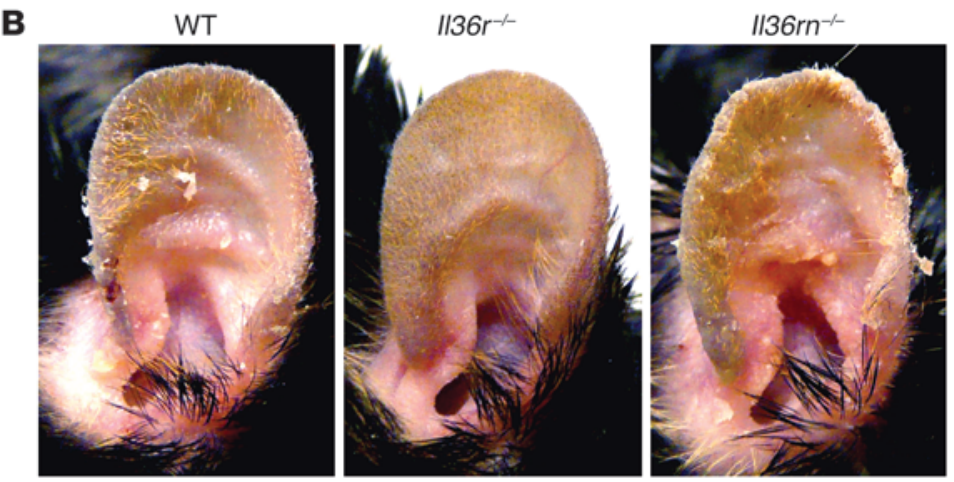

C
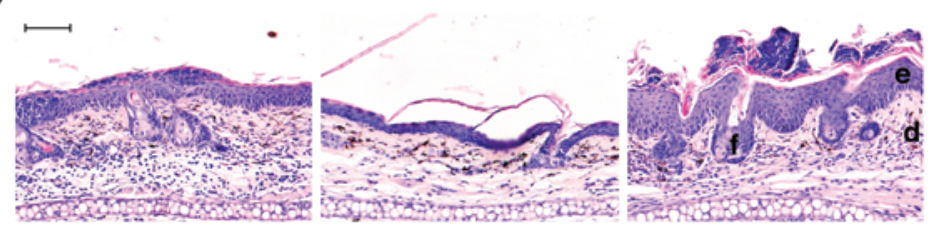

\section{Figure 1}

IL-36R and IL-36R antagonist play critical roles in the development of IMQ-induced psoriasiform dermatitis. Ears of both $/ / 36 \mathrm{r}^{-1-}$ and $/ / 36 \mathrm{rn} \mathrm{H}^{-/-}$mice and WT mice ( $n \geq 4$ per group) were topically treated with an IMQ-containing cream (Aldara, MEDA Pharma) for 7 consecutive days. (A) Ear swelling was measured daily before treatment. Values show averages \pm SEM. ${ }^{\star} P<0.05$; ${ }^{\star *} P<0.01 ;{ }^{\star * \star} P<0.001$. (B) At day 7 , photographs were taken from whole ears or (C) H\&E-stained ear sections representing the thickest epidermal region of each ear from individual mice representative of indicated groups. Epidermis (e), dermis (d), and hair follicle (f) are indicated. Data are representative of 4 experiments. Scale bar: $100 \mu \mathrm{m}$.

canoylphorbol-13-acetate and induced skin pathology reminiscent of human psoriasis (32). Moreover, removal of the IL36RN gene in K14-IL-36 $\alpha$ transgenic mice induced chronic and aggravated skin abnormalities, and mutations in IL36RN were recently described in patients with pustular psoriasis $(33,34)$. IL-36 expression in keratinocytes was enhanced by IL- $1 \alpha$, TNF- $\alpha$, and IL-17 and downregulated by neutralizing IL-22 (29). IL-17 induced IL-36 agonists more potently in human psoriasis-derived keratinocytes than in healthy keratinocytes, while Il36rn expression remained unaffected (35). Furthermore, IL-36 was able to induce its own expression as well as expression of various proinflammatory cytokines and augmented IL-17-mediated production of antibacterial peptides $(29,36)$.

IL-36 is thought to act mainly in an autocrine fashion on skin-resident cells like keratinocytes and fibroblasts, which express high amounts of IL-36 and IL-36R. However, lower levels of IL-36R are also expressed on bone marrow-derived DCs (BMDCs) and $\mathrm{CD}^{+} \mathrm{T}$ cells. Consistently, when treated with superactive forms of IL-36, DCs upregulated CD80, CD86, and MHC class II and produced proinflammatory cytokines like IL-12, IL-1 $\beta$, TNF- $\alpha$, and IL-23 (37).

Clearly, several feedback loops exist in the regulatory circuits of psoriasis. But the individual contributions of the different cytokines and their relative impact on skin pathology are not well understood. In this work, we show that IL-36 cytokines are master regulators of the IL-23/IL-17/IL-22 pathway and development of cutaneous pathology in response to IMQ.

\section{Results}

$I L-36 R$ is essential for the development of IMQ-induced psoriasis. We investigated the development of IMQ-induced psoriasiform dermatitis in IL-36R-deficient (Il36r/-) mice by topical treatment of ear skin with an IMQ-containing cream (Aldara). As shown in Figure 1, Il36r/- mice were protected from the pathological manifestations of psoriasis, including ear swelling, acanthosis, erythema, skin flaking, and hyperkeratosis. To assess the role of IL-36RN in this disease model, we generated IL-36R antagonist-deficient (Il36rn-/-) mice (Supplemental Figure 1; supplemental material available online with this article; doi:10.1172/ JCI63451DS1) and treated them with Aldara. Consistent with the results above, mice lacking IL-36RN showed increased severity of key disease features (Figure 1).

IL-36 regulates the recruitment of inflammatory cells and the expansion of IL-17A-producing $\gamma \delta T$ cells in the skin. To further characterize the cellular and molecular pathways affected by IL-36, we characterized and quantitated the inflammatory ear infiltrate by flow cytometry. The lesions of WT mice showed a remarkable infiltration of $\mathrm{CD}^{4} 5^{+}$leucocytes, consisting mainly of neutrophils and macrophages, which was absent in $\mathrm{Il36r}^{-/-}$mice and 3-fold enhanced in Il36rn-/- mice compared with that in WT mice (Figure 2, A-D). $\gamma \delta$ T cells showed a much more pronounced expansion than $\alpha \beta$ T cells and accounted for the majority of T cells in the affected ear during the course of disease in WT mice (Figure 2, $\mathrm{E}$ and $\mathrm{F})$. The expanded $\gamma \delta \mathrm{T}$ cells were mainly composed of dermal $\gamma \delta$ TCR $^{\text {lo }}$ cells, but not $\gamma \delta$ TCR $^{\text {hi }}$ dendritic epidermal T cells, and the former were by far the most prominent IL-17A producers (Figure 2I). Notably, $\gamma \delta \mathrm{T}$ cells and consequently IL-17A-producing cells failed to expand in Il36r-1- mice (Figure 2, E, G, and H), while $\alpha \beta$ T cells remained virtually unaffected by the loss of IL-36R (Figure 2F). Characterization of the few IL-17A producers in Il36r/mice showed that they were also mainly composed of $\gamma \delta \mathrm{T}$ cells (Figure 2J), indicating that absence of IL-36R signaling affected primarily the expansion of $\gamma \delta \mathrm{T}$ cells and to a lesser extent their propensity to produce IL-17A (Figure $2 \mathrm{~K}$ ). Il36 $\mathrm{rn}^{-/-}$mice showed no differences in expansion of $\gamma \delta \mathrm{T}$ cells and IL-17A-producing cells. The changes observed in the skin infiltrates were partially 

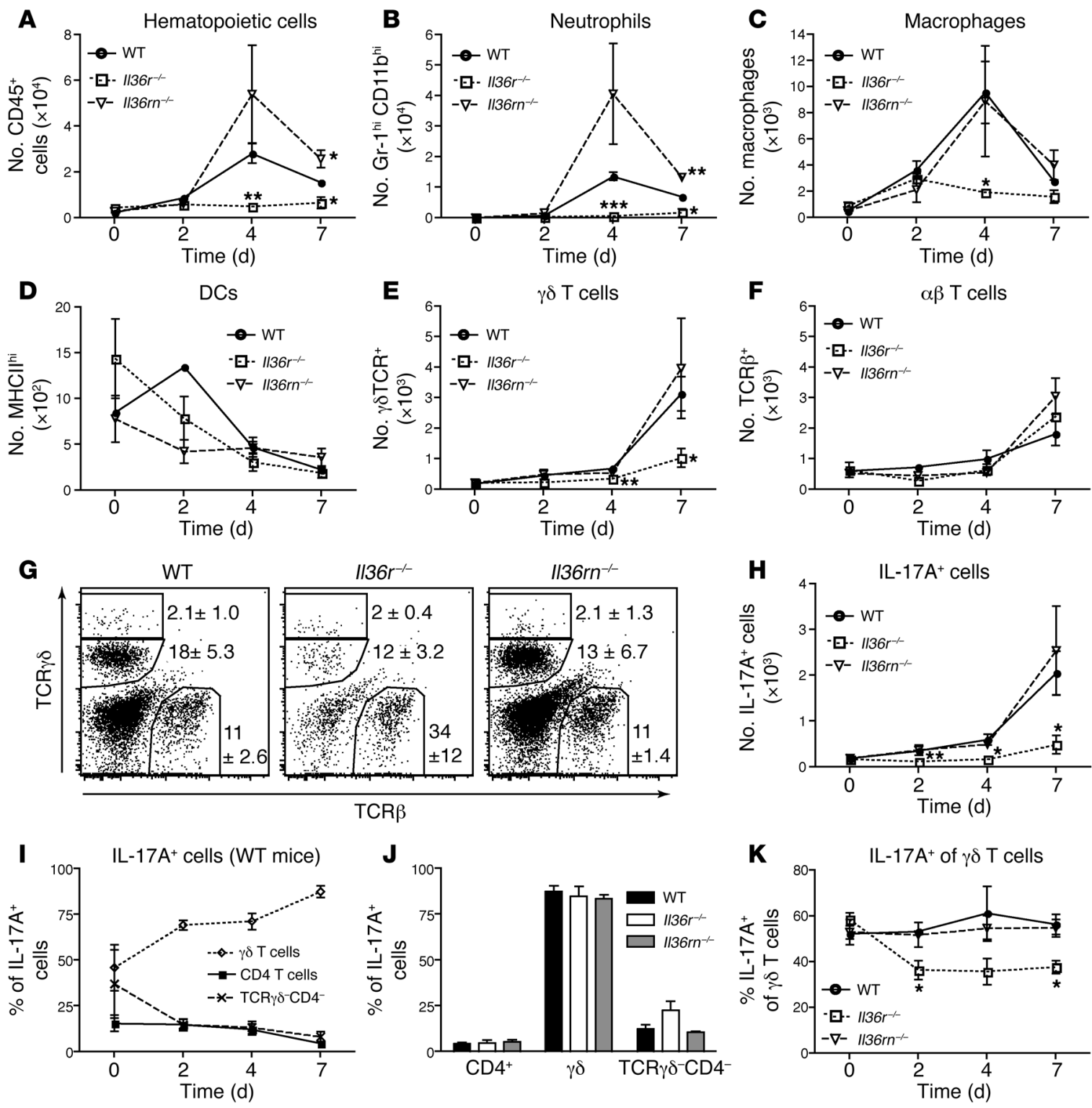

\section{Figure 2}

IL-36 regulates the recruitment of inflammatory cells and the expansion of IL-17A-producing $\gamma \delta \mathrm{T}$ cells in the skin. Mice were treated as described in the legend to Figure 1. At days indicated, mice ( $n=3 /$ group) were sacrificed and immune cells in the ear were characterized by flow cytometry. Graphs

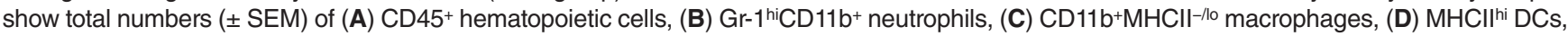
(E) $\gamma \delta$ T cells, and $(\mathbf{F}) \alpha \beta$ T cells per ear. (G) Dot plots show expression of TCR $\gamma \delta$ and TCR $\alpha \beta$ on CD $45^{+}$cells. The TCR $\gamma \delta^{h i}$ population represents dendritic epidermal T cells. Values represent average percentages \pm SD of the gated populations from groups of mice. (H) Total numbers of IL-17Aproducing cells in the ears at indicated days. (I) Characterization of cell types producing IL-17A in WT mice throughout the course of IMQ treatment. (J) Percentages of CD4+ T cells, $\gamma \delta$ T cells, and CD4- $\gamma \delta^{-}$cells among IL-17A producers in ears of indicated groups of mice at day 7 after treatment. (K) Percentages of IL-17A producers among $\gamma \delta \mathrm{T}$ cells in ears of indicated groups of mice. Data are representative of 4 independent experiments. (H-K) Values show averages \pm SEM. ${ }^{\star} P<0.05 ;{ }^{* \star} P<0.01 ;{ }^{* \star \star} P<0.001$

mirrored in the draining lymph nodes (dLNs). During the course of disease, we observed a reduction in cellularity and frequencies of neutrophils and macrophages in Il36r/- mice, albeit this decrease was not as strong as that in the ears (Supplemental Figure 2, A-C). Similar to the skin, $\gamma \delta$ but not $\alpha \beta$ T cells expanded vigorously and were the main producers of IL-17A (Supplemental Figure 2, D-G). However, IL-17A-producing $\gamma \delta$ T cells were only slightly reduced in dLNs of Il36r/- mice (Supplemental Figure 2, D and F). These results demonstrate that IL-17 is primarily derived from dermal $\gamma \delta$
$\mathrm{T}$ cells, in agreement with recent reports $(38,39)$, and that IL-36R is indispensable for expansion of pathogenic IL-17-producing $\gamma \delta$ $\mathrm{T}$ cells and development of skin disease.

IL-36R on radioresistant skin-resident cells is crucial for psoriasis. IL-36R is predominantly expressed in epithelial tissues, including the skin (36), but it has also been detected in DCs and T cells (37). To distinguish the importance of IL-36R on nonhematopoietic skin-resident cells compared with that on radiosensitive hematopoietic cells in disease development, we generated recipro- 

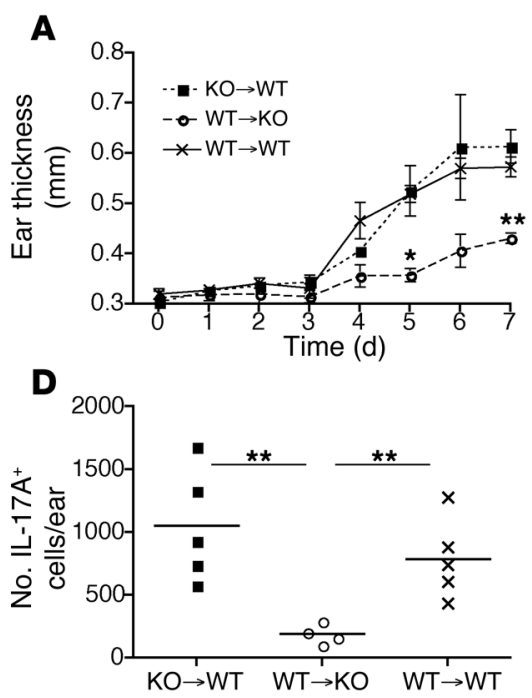
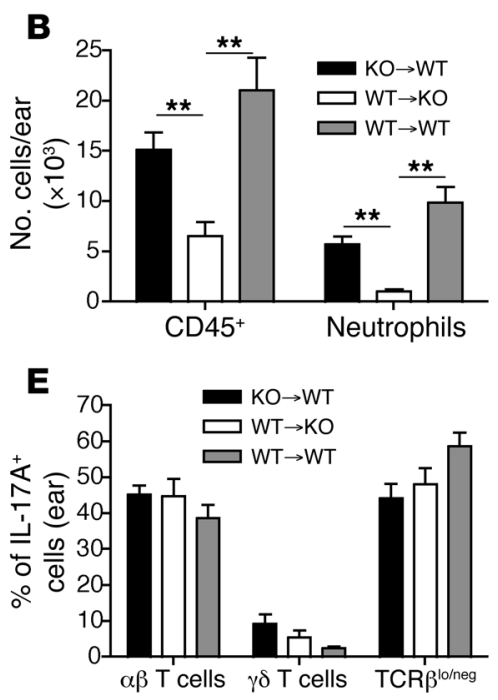
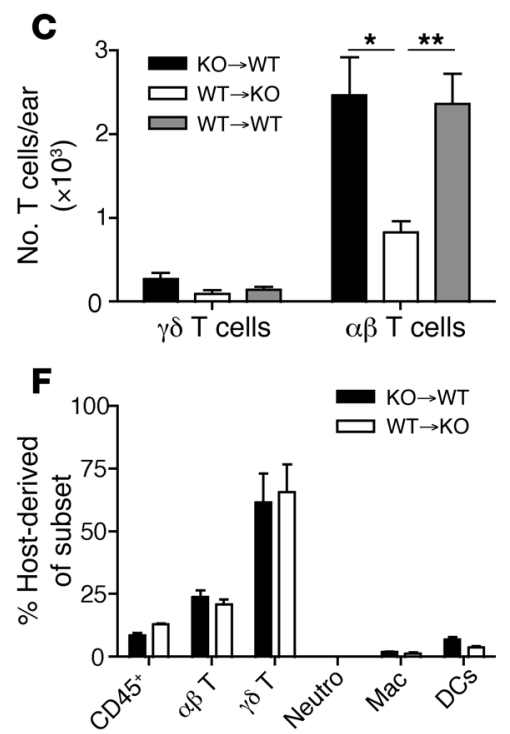

\section{Figure 3}

IL-36R on radioresistant resident cells is crucial for the expression of IL-17A in the skin and development of IMQ-induced skin inflammation. Reconstituted bone marrow chimeras, $/ / 36 r^{-1-} \mathrm{CD} 45.2 \mathrm{BM} \rightarrow \mathrm{WT}$ CD45.1 mice $(\mathrm{KO} \rightarrow \mathrm{WT})$, WT CD45.1 BM $\rightarrow / / 36 r^{-1-} \mathrm{CD} 45.2 \mathrm{mice}(\mathrm{WT} \rightarrow \mathrm{KO})$, and WT CD45.1 BM $\rightarrow$ WT CD45.1 mice (WT $\rightarrow$ WT), were treated with Aldara cream, as described in the legend to Figure 1. (A) Ear thickness was monitored daily. (B-F) At day 7, cell populations in the ear were characterized by flow cytometry. Total numbers of (B) CD45+ hematopoietic cells and neutrophils, (C) $\gamma \delta$ and $\alpha \beta$ T cells, and (D) IL-17A-producing cells in ears of indicated chimeras are shown. (E) Characterization of IL-17A+ cells. (F) Percentage of host-derived remaining hematopoietic cell populations that survived the irradiation and were not replaced. (A-C, E, and $\mathbf{F})$ Values indicate averages \pm SEM of groups. (D) Symbols represent individual mice, and horizontal lines indicate averages of groups. ${ }^{\star} P<0.05 ;{ }^{\star \star} P<0.01$.

cal bone marrow chimeras. Lethally irradiated C57BL/6 mice were reconstituted with $\mathrm{Il36r^{-/ }}$ bone marrow $\left(\mathrm{CD} 45.2^{+} \mathrm{KO} \rightarrow \mathrm{CD} 45.1^{+}\right.$ $\mathrm{WT})$ and vice versa $\left(\mathrm{CD} 45.1^{+} \mathrm{WT} \rightarrow \mathrm{CD} 45.2^{+} \mathrm{KO}\right) . \mathrm{CD} 45.1^{+}$ $\mathrm{WT} \rightarrow \mathrm{CD} 45.1^{+}$WT chimeras served as controls. We found that the expression of IL-36R on radioresistant skin-resident cells, but not on hematopoietic bone marrow-derived cells, was essential for pathology upon IMQ treatment (Figure 3A). Accordingly, the swelling and the cutaneous inflammatory response, in particular neutrophil recruitment, were impaired in the absence of IL-36R on skin-resident cells, while mice lacking IL-36R on radiosensitive leukocytes showed swelling and an inflammatory response analogous to that of WT $\rightarrow$ WT mice (Figure 3, A and B). Similarly, recruitment of neutrophils into dLNs was affected in the absence of IL-36R on nonhematopoietic skin-resident cells (data not shown). Detailed analysis of $\mathrm{T}$ cell populations in the ears showed an up to $90 \%$ drop in numbers of dermal $\gamma \delta$ T cells in all 3 groups of chimeras when compared with those in nonirradiated mice (Figure 2, E and G, and Figure 3C). This indicates that in nonirradiated WT mice a fetal-derived radiosensitive $\gamma \delta \mathrm{T}$ cell subset expanded in response to IMQ and is responsible for the IL-17 production. Interestingly, the $\alpha \beta$ T cell expansion observed in the irradiated chimeras was dependent on IL-36R expression by skin-resident cells (Figure 3C), while it was independent of IL-36R in nonirradiated mice (Figure $2 \mathrm{~F}$ ). In addition, IL-17A production in the skin lesions of the WT $\rightarrow$ WT chimeras was found in $\mathrm{TCR} \beta^{\text {hi }}$ and TCR $\beta^{\text {lo }} \alpha \beta$ T cells, which were reduced in WT $\rightarrow \mathrm{KO}$ chimeras but not in $\mathrm{KO} \rightarrow \mathrm{WT}$ chimeras (Figure 3, D and E). Staining of CD45.1 (WT) and CD45.2 (KO) cells enabled us to verify that most $(>90 \%)$ of the $\mathrm{CD} 45^{+}$cells in ear skin of the chimeras originated from donor bone marrows (Figure 3F). Interestingly, the few $\gamma \delta$ T cells present in the skin of chimeras were derived primarily from the host, but they played a minor role, as their numbers were limited relative to IL-17A-producing $\alpha \beta \mathrm{T}$ cells. Together, these results demonstrate that the expression of IL-36R by skin-resident cells, such as keratinocytes and fibroblasts, is pivotal for the cutaneous pathology.

$I L-17^{+} \alpha \beta T$ cells can partially mediate psoriasiform disease in the absence of $I L-17^{+} \gamma \delta T$ cells. Previous reports suggested that IL-23 promotes the expansion of pathogenic IL-17-producing $\gamma \delta \mathrm{T}$ cells $(38,39)$. Surprisingly, we found that in $\mathrm{Tcrd}^{-/-}$mice ear swelling was only moderately inhibited and that the inflammatory infiltrate was insignificantly reduced (Figure 4, A and B), despite a substantial decrease in the number of IL-17A-producing cells (Figure 4C). Notably, $\alpha \beta \mathrm{T}$ cells and a minor population of non- $\mathrm{T}$ cells accounted for the remaining IL-17A production in $\mathrm{Tcrd}^{-/-}$mice (Figure 4D), corroborating data in WT $\rightarrow$ WT chimeras, which lacked dermal $\gamma \delta$ T cells. Taken together, our results suggest that IL-17-producing $\alpha \beta$ T cells (Th17 cells) can partially compensate for the absence of dermal $\gamma \delta$ T cells as mediators of cutaneous pathology.

IL-36 mediates cutaneous pathology mainly but not only by induction of the IL-23/IL-17/IL-22 pathway. In the light of the above results, we reassessed the roles of the IL-23/IL-17/IL-22 pathway by comparing the respective knockouts with each other and with Il36r ${ }^{-1-}$ mice in the IMQ model. Expectedly, both $I l 23 \mathrm{a}^{-/-}$and $I l 17 a^{-/-}$mice showed impaired disease development, although the $I l 23 \mathrm{a}^{-/-}$mice were better protected. Skin flaking and erythema were visibly reduced in $I l 17 a^{-/-}$mice and almost undetectable in $\mathrm{Il} 23 \mathrm{a}^{-/-}$mice (data not shown). Accordingly, $I l 17 \mathrm{a}^{-/-}$and, in

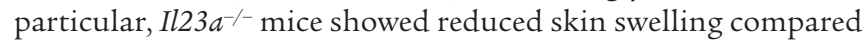
with that of WT mice (Figure 5A). Cellular analysis revealed that neutrophilia in the ears was only partially dependent on IL-17A 

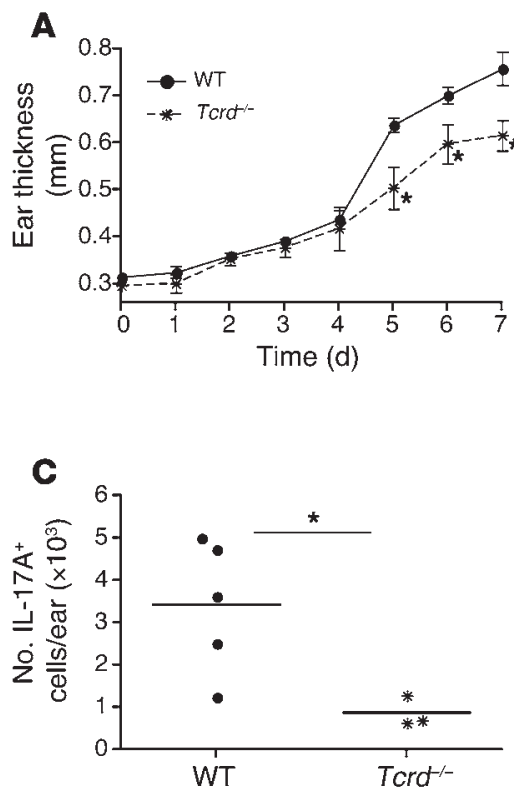

\section{B}
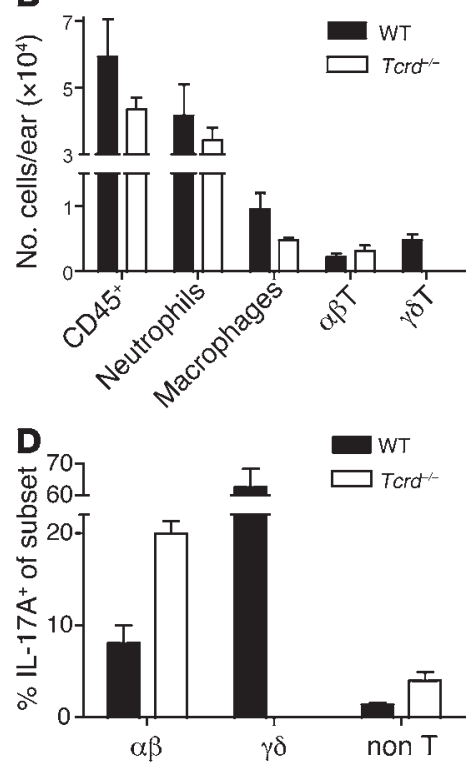

\section{Figure 4}

IL-17A production and skin disease can be partially mediated by $\alpha \beta$ T cells in $\gamma \delta$ T cell-deficient mice. Mouse ears were treated with Aldara as described above. (A) Ear thickness was monitored daily. Values show averages \pm SEM $(n \geq 3)$. (B-D) At day 7 , cell populations in the ear were characterized by flow cytometry. (B) Total numbers of indicated populations and (C) IL-17A-producing cells per ear are shown. Symbols represent individual mice, and horizontal lines indicate averages of groups. Values indicate averages \pm SEM. (D) Percentages of $\alpha \beta$ T cells, $\gamma \delta$ T cells, and non-T cells among IL-17A-producing cells in ears. ${ }^{*} P<0.05$. expression, while it was completely abrogated in the absence of IL-23 (Figure 5B). IL-23 deficiency almost completely prevented the expansion of $\gamma \delta \mathrm{T}$ cells, including the IL-17A producers (Figure 5, C and D). The few remaining $\gamma \delta \mathrm{T}$ cells showed a reduced propensity to produce IL-17A (Figure 5E). In addition, IL-17A-producing $\alpha \beta$ T cells were reduced in the skin and dLNs of Il23 $a^{-1-}$ mice (Figure 5, E and F). We reasoned that the remaining neutrophil influx and skin swelling observed in $I l 17 \mathrm{a}^{-/-}$mice was due to the presence of other pathogenic effector cytokines such as IL-22. Indeed, ear thickness and skin neutrophilia were similarly reduced in $I l 22^{-/-}$and $I l 17 a^{-/-}$mice (Figure 5, G and H), suggesting similar effector activities elicited by the 2 cytokines. The number of IL-17A-producing cells was slightly increased in $I l 22^{-/-}$mice compared with that in WT mice, possibly indicating some compensatory mechanisms (Figure 5I). Regardless, skin pathology was more severe in $I l 17 a^{-/-}$and $I l 22^{-/-}$mice than in Il36r/- mice. To assess the combined effects of IL-17 and IL-22, we inhibited IL-22 in $I l 17 a^{-/-}$mice using neutralizing antibodies (Figure 5, J-L). In this case, most effector functions and pathological manifestations of the disease were gone. Skin swelling was similarly reduced in $I l 22^{-/-}$mice and WT mice treated with anti-IL-22 mAb, confirming efficient neutralization of IL-22 in the latter (data not shown). Nevertheless, in comparison with Il36r $\mathrm{r}^{-1}$ mice, residual ear swelling could be observed (Figure 5J). Together, these experiments suggest that IL-36 drives skin lesion development mainly by induction of the IL-23/IL-17/IL-22 axis, but it can promote skin thickening to a minor extent in the absence of these cytokines.

$I L-36$ promotes $I L-17$ A production in the skin and IMQ-induced psoriasis independently of IL-1R signaling. Considering that IL-23-mediated expansion of IL-17A-producing $\gamma \delta \mathrm{T}$ cells has been reported to depend on IL-1 (38), and IL-36 $\alpha$ overexpression in the skin promotes expression of inflammatory mediators including IL-1 $\alpha$ (31), we reasoned that IL-1 and IL-23 might be downstream of IL-36 and cooperate in IMQ-driven psoriasis. However, IL-1R1 deficiency did not protect mice from IMQ-induced psoriasiform dermatitis. Ear swelling, acanthosis, and skin flaking were comparable in $I l 1 \mathrm{rl}^{-/}$ and WT mice (Figure 6, A and B). Furthermore, IL-17A-producing T cells expanded normally in ears of $\mathrm{Illr}_{11^{-/-}}$mice (Figure 6, D-F). Nevertheless, recruitment of neutrophils was considerably reduced, although not to the level of that in $\mathrm{Il36r}^{-1}$ mice (Figure 6C). Thus, IL-1R signaling is important for neutrophil influx but redundant for the development of IL-17A-producing $\gamma \delta \mathrm{T}$ cells and the pathology of IMQ-induced psoriasiform dermatitis.

IL-36 promotes expression of itself along with chemokines and keratinocyte mitogens in dermal mesenchymal cells and keratinocytes. Since IL-36R was primarily required on skin-resident cells but not on hematopoietic cells for disease development (Figure 3), we wanted to analyze the direct effects of IL-36 and IMQ on nonhematopoietic skin-resident cells. Therefore, we sorted $\mathrm{CD} 45^{-}$cells from either epidermis or dermis. The majority of epidermal CD $45^{-}$cells are keratinocytes, whereas dermal $\mathrm{CD}^{4} 5^{-}$cells consist predominantly of mesenchymal cells (fibroblasts, adipocytes, and vascular cells). These cell populations were cultured with IMQ and super-

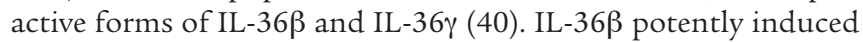
expression of IL-36 cytokines, the neutrophil- and T cell-attracting chemokines CXCL1 and CCL20, and the keratinocyte mitogens G-CSF and TGF- $\alpha$ (Figure 7A). Similar results were obtained using IL-36 $\gamma$ for stimulation (data not shown). In contrast, IMQ showed no such activity (Figure 7A), consistent with the absence of TLR7 expression on CD45- dermal mesenchymal cells and keratinocytes (Figure 7B).

$D C$ s are crucial for the induction of IMQ-driven psoriasis. Considering the abundance of TLR7 expression on DCs and its absence on CD45- keratinocytes and dermal mesenchymal cells (Figure 7B), we speculated that DCs are the main sensors of IMQ and producers of IL-23. To assess the role of DCs in the IMQ psoriasis model, we injected diphtheria toxin into CD11c-DTR mice, which effectively depleted all CD $11 c^{+}$DCs until analysis of mice at day 4 after IMQ treatment (Figure 8B). Absence of DCs protected mice from ear swelling, neutrophil infiltration, $\mathrm{T}$ cell expansion, and development of IL-17-producing cells (Figure 8, A and C-E). Notably, stimulation of BMDCs with IMQ or IL-36 $\beta$ induced expression of $I l 23 a$ as well as $I l 36 a$ and $I l 36 g$ (Figure $8 F$ ). 

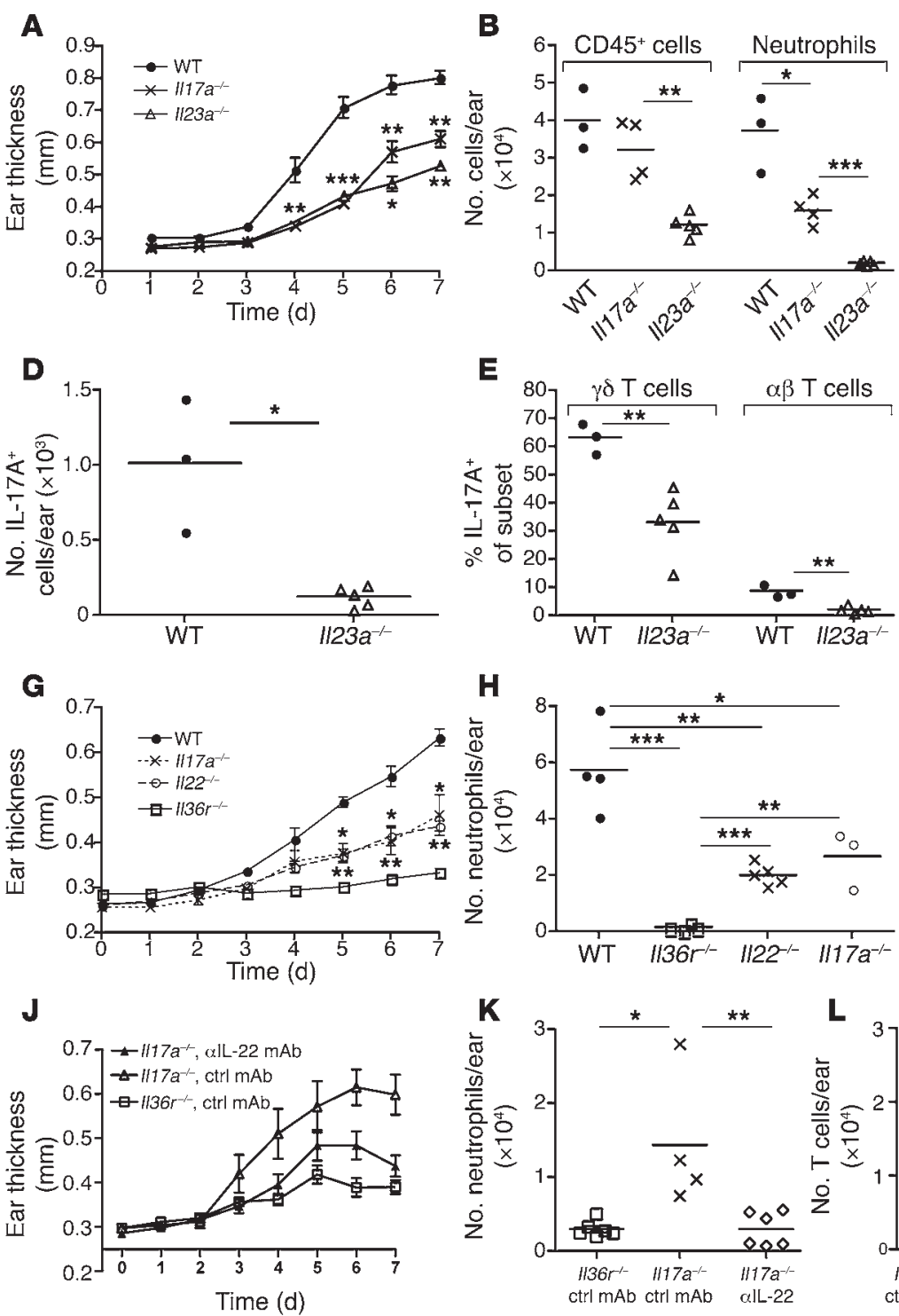
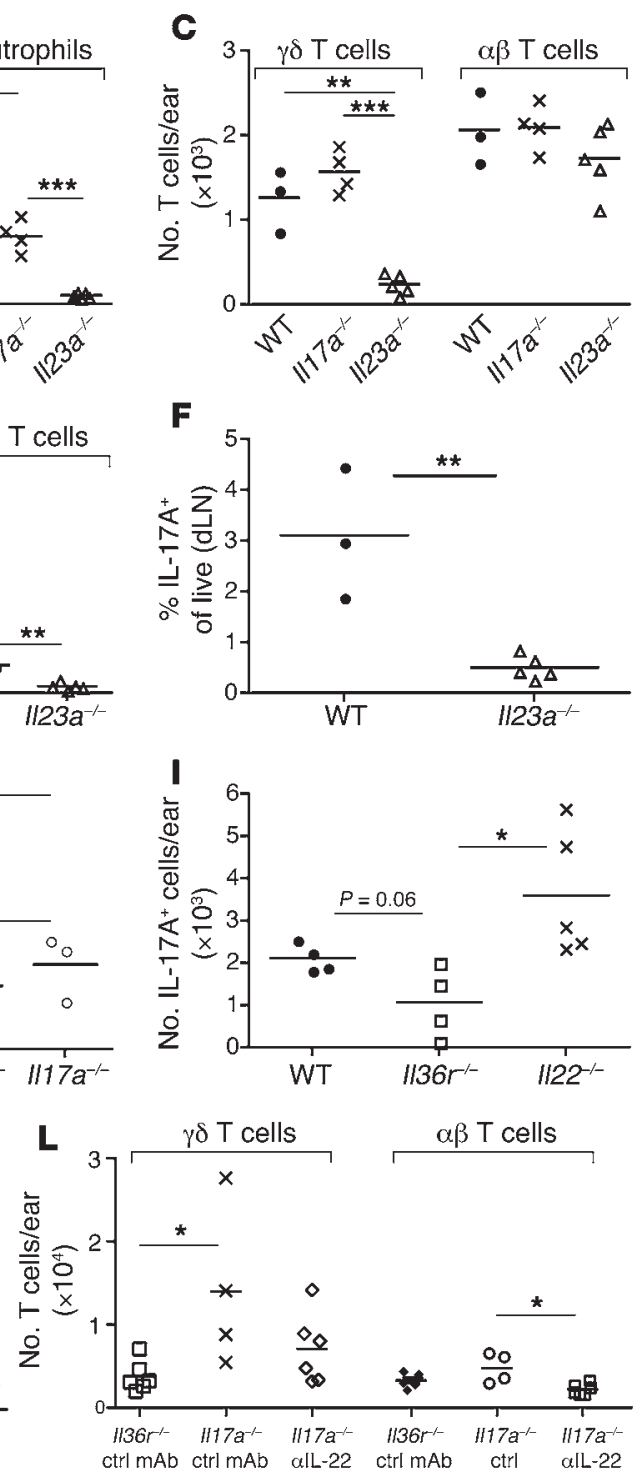

\section{Figure 5}

$1 / 36 r^{-1-}$ mice are better protected than mice lacking IL-23, IL-17A, or IL-22. (A, G, and $\mathbf{J}$ ) Ears of indicated groups of mice were treated with Aldara, and ear thickness was analyzed as described above. Values show averages \pm SEM. Cell populations in the ear were characterized by flow cytometry at day 7. Total numbers of (B, H, and $\mathbf{K})$ CD45+ hematopoietic cells and neutrophils, (C and $\mathbf{L}) \gamma \delta$ and $\alpha \beta$ T cells, and (D and I) IL-17A-producing cells in ears of indicated mice. (E) Percentage of IL-17A producers among $\gamma \delta$ and $\alpha \beta$ T cells in ear lesions. (F) Percentage of IL-17A-producing cells gated on lymphocytes in dLNs. (J-L) I/17a-/- mice treated with neutralizing anti-IL-22 or control (ctrl) mAb compared with $1 / 36 r^{-1-}$ mice treated with control mAb. (B-F, H, I, K, and $\mathbf{L}$ ) Symbols represent individual mice, and horizontal lines indicate averages of groups. ${ }^{\star} P<0.05 ;{ }^{* \star} P<0.01 ;{ }^{* *} P<0.001$.

\section{Discussion}

Current evidence implicating IL-36 cytokines in the development of psoriasis is mainly based on transgenic mice that develop dermatitis due to forced overexpression of IL-36 $\alpha$ in the epidermis $(31,32)$ and the recent identification of IL-36R antagonist loss-offunction mutations in patients with a rare and very severe form of psoriasis termed generalized pustular psoriasis $(33,34)$. To our knowledge, our study demonstrates for the first time that IL-36R signaling is absolutely crucial for control of the pathogenic IL-23/IL-17/IL-22 axis and development of psoriasiform dermatitis in response to environmental cues triggering TLR7 on skin DCs (i.e., using topical application of IMQ). IL-36R knockouts were (completely) protected from IMQ-induced skin pathology observed in WT mice, including hyperkeratosis, acanthosis, neutrophil recruitment, and expansion of IL-17-producing T cells. Moreover, mice lacking IL-36R antagonist showed exacerbated disease. Together, these data suggest that the balance of endogenous IL-36R ligands and IL-36R antagonist plays a pivotal role in IMQ-induced skin disease.

IMQ treatment of WT mice resulted in vigorous expansion of IL-17-producing dermal $\gamma \delta \mathrm{T}$ cells that are mainly responsible for the psoriasiform disease, as suggested recently (38). However, we also found that $\alpha \beta$ T cells can contribute substantially to cutaneous pathology and inflammation when $\gamma \delta \mathrm{T}$ cells are 
A
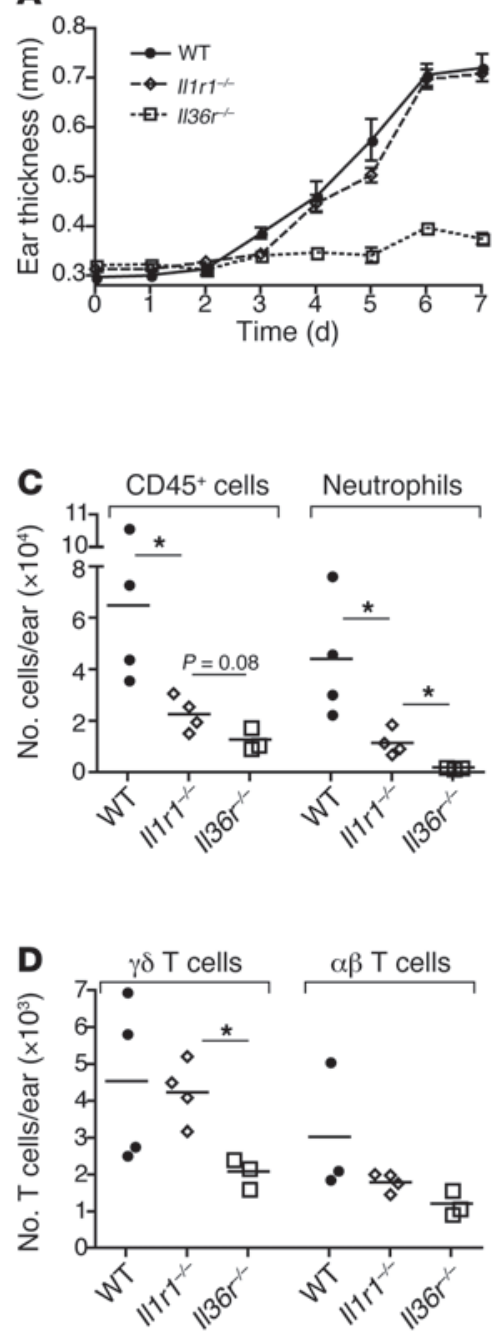

B

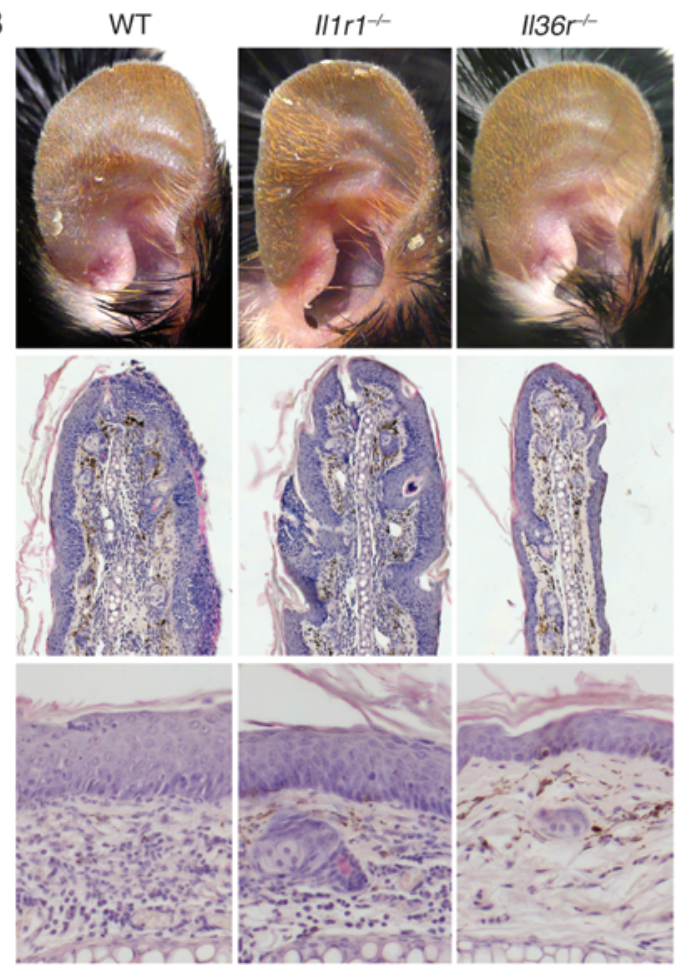

E

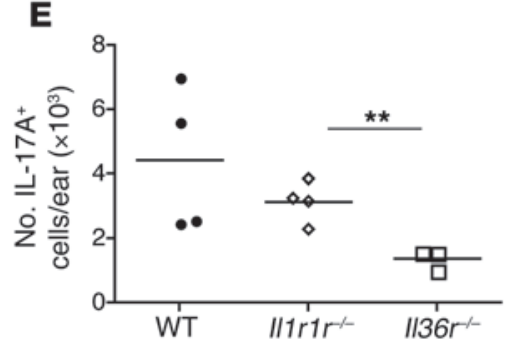

\section{Figure 6}

IL-36 promotes IL-17A expression in the skin and IMQ-induced psoriasis independent of IL-1R1 signaling. $/ / 1 r 1^{-/-}, / / 36 r^{-1}$, and WT mice were treated with Aldara, as described in the legend to Figure 1. (A) Ear thickness was monitored daily. Values show averages \pm SEM $(n \geq 3)$. (B) At day 7, photographs were taken from whole ears (top row) and H\&E-stained ear sections, representing the ear tip (middle row) and the thickest epidermal region (bottom row) from individual mice representative of indicated groups. Scale bar: $100 \mu \mathrm{m}$. On day 7 , cells prepared from ears were analyzed by flow cytometry. The graphs show the total numbers of $(\mathbf{C})$ infiltrating CD45+ hematopoietic cells and neutrophils, (D) $\alpha \beta$ and $\gamma \delta$ T cells, and (E) IL-17A-producing cells per ear. (C-E) Symbols represent individual mice, and horizontal lines indicate averages of groups. (F) Dot plots showing IL-17A-producing cells and $\gamma \delta \mathrm{T}$ cells. Values indicate frequencies of IL-17+ $\gamma \delta^{+} \mathrm{T}$ cells in adjacent gated fields. ${ }^{*} P<0.05$; ${ }^{\star \star} P<0.01$.

$\mathbf{F}$

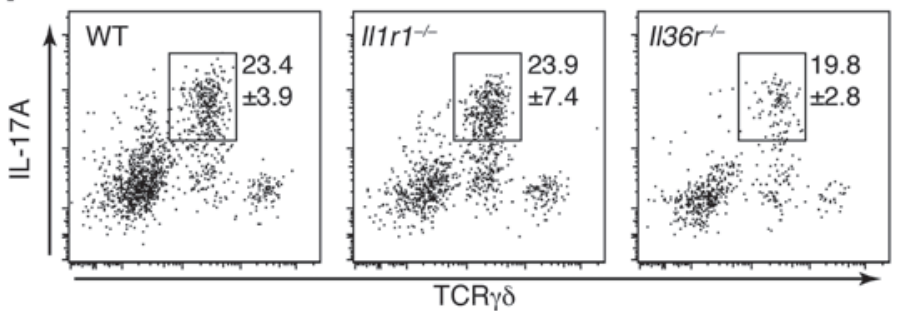

absent. TCR $\delta$-deficient mice showed only a moderate reduction in ear swelling and no differences in neutrophilia, probably due to a 2 -fold increase in frequencies of Th17 cells compared with WT mice. Interestingly, in concordance with a recent report showing that dermal $\gamma \delta \mathrm{T}$ cells are radiosensitive and not reconstituted after transfer of adult bone marrow upon irradiation (41), we found that Th17 cells but not $\gamma \delta \mathrm{T}$ cells expanded in the skin upon IMQ treatment of WT $\rightarrow$ WT chimeras. Thus, Th17 cells can compensate, at least in part, for the absence of $\gamma \delta \mathrm{T}$ cells in mice, indicating functional plasticity. This is interesting in the context of human skin in which $\alpha \beta \mathrm{T}$ cells are far more prominent than $\gamma \delta \mathrm{T}$ cells, although presence of the latter has also been described in psoriatic lesions (38). Regardless, our data demonstrate that IL-36R signaling was essential for the expansion of both IL- $17^{+} \gamma \delta$ T cells and IL- $17^{+} \alpha \beta$ T cells (in the absence of $\gamma \delta \mathrm{T}$ cells).

As mentioned above, there is overwhelming evidence for a pivotal role of the IL-23/IL-17/IL-22 axis in development of disease, with IL-23 driving development of pathological IL-17- and IL-22-producing T cells in the skin. Our analysis of mice lacking IL-23, IL-17, or IL-22 confirmed a pathological effector function of these cytokines but also revealed interesting differences, espe-

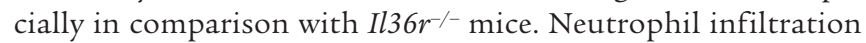
and ear swelling were comparably reduced $(\sim 60 \%)$, but not abrogated, in $\mathrm{Il} 22^{-/-}$and $\mathrm{Il} 17 \mathrm{a}^{-/-}$mice, suggesting that both cytokines exert important but partially redundant effector functions in the 

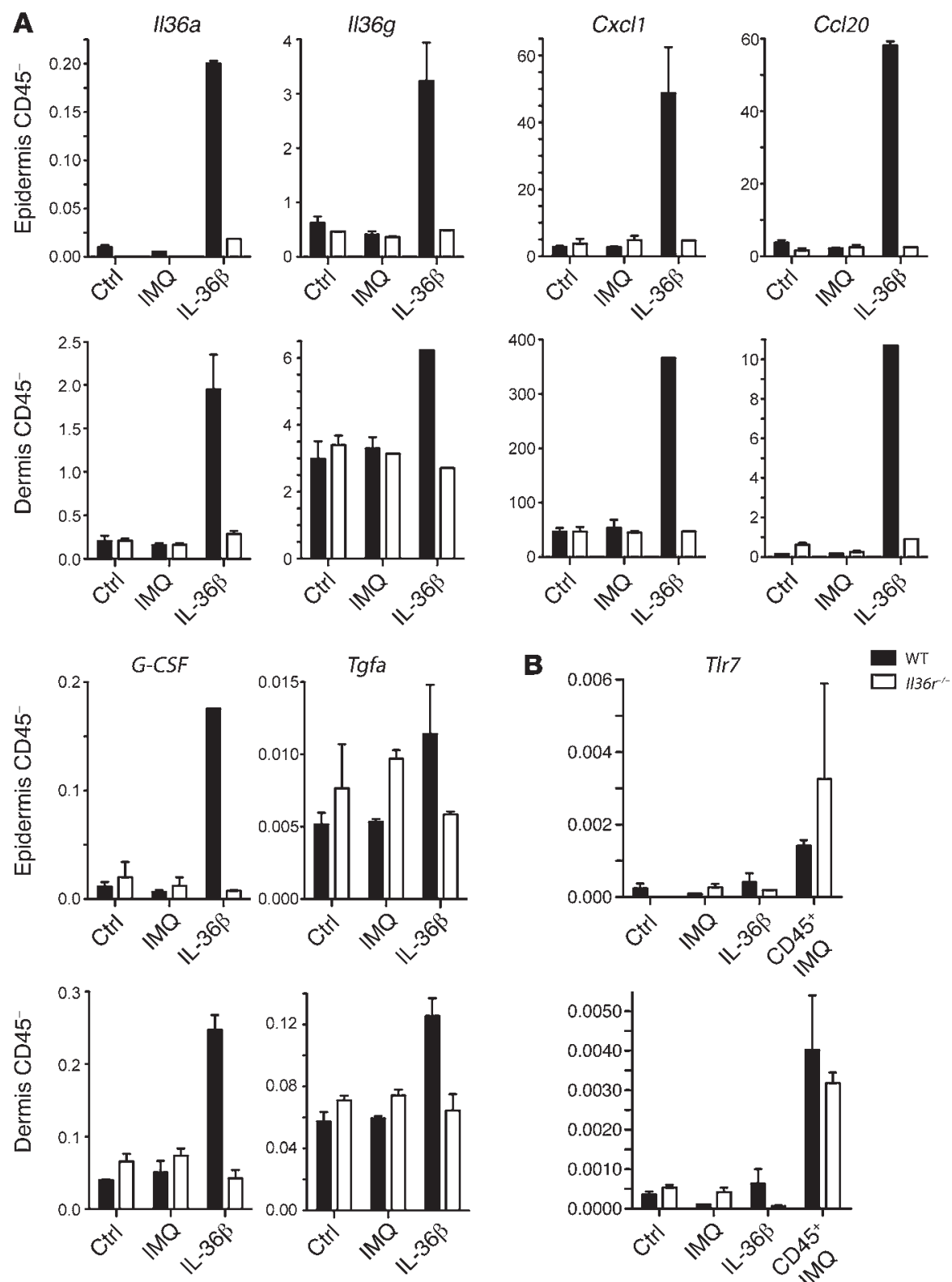

\section{Figure 7}

Autocrine IL-36 production by skin-resident cells promotes expression of chemokines and growth factors involved in disease manifestation. Ear dermis and epidermis were separated. CD45+ hematopoietic cells and CD45- dermal and epidermal cells, consisting almost exclusively of dermal mesenchymal cells and epidermal keratinocytes, respectively, were purified (>99\%) by cell sorting. CD45- dermal and epidermal cells were cultured for 8 hours in the absence (Ctrl) or presence of IMQ or recombinant IL-36 $\beta$. (A) Expression of indicated genes was measured by realtime PCR. (B) TIr7 expression in CD45+ and CD45- cells from dermis and epidermis stimulated as indicated. Values show mean \pm SEM normalized to G6PD. development of IMQ-induced lesions. Indeed, IL-17 and IL-22 have been shown to cooperate additively in the upregulation of antimicrobial peptides, including $\beta$-defensin- 2 and S100A9 in keratinocytes (42). Consistently, neutralization of IL-22 in $I l 17 a^{-/-}$ mice inhibited skin swelling and neutrophil recruitment more than the absence of either cytokine (Figure 5, J-L). In line with previous reports demonstrating a critical role of IL-23 in psoriasis mouse models $(7,10,11,14,15)$ and in human psoriasis (23-26), IMQ-treated $I l 23 \mathrm{a}^{-/-}$mice showed a striking reduction in skin swelling, concomitant with the absence of neutrophils and IL-17 ${ }^{+} \gamma \delta$ T cells (Figure 5, B-E) and Il22 mRNA levels (43). Nevertheless, absence of IL-23 or both IL-17 and IL-22 resulted in a measurable, increased skin swelling compared with $\mathrm{Il36r^{-1 }}$ mice (Figure 5). These results suggest that IL-36 contributes to a small but not negligible degree to lesion development beyond induction of IL-23/IL-17/IL-22 axis in the IMQ psoriasis model. Notably, 12-O-tetradecanoylphorbol-13-acetate treatment of K14-IL-36 $\alpha$ transgenic mice induced psoriasiform dermatitis also on a lymphocyte-deficient rag2 $2^{-/-}$background, indicating that $\mathrm{T}$ cells and their pathological mediators, IL-17 and IL-22, are completely dispensable in this transgenic mouse model (32).

IL-36 has also been shown to upregulate IL-1 $\alpha$ expression $(31,37)$, which can support keratinocyte survival and proliferation through the induction of FGF7 and GM-CSF in fibroblasts (44). Moreover, IL-1 together with IL-23 is essential for expansion of IL-17-producing $\gamma \delta \mathrm{T}$ cells $(22,38)$. This led us to hypothesize that IL-36 mediates IMQ psoriasis through the induction of IL-1. Indeed, IL-1 has also been believed to play a pivotal role in cutaneous pathology in mice and humans. IL-1Ra deficiency (45-47) or transgenic overexpression of IL-1 $\alpha$ in keratinocytes (8) results in severe cutaneous inflammation similar to transgenic overexpression of IL-36 $\alpha$. However, our data revealed that epidermal hyperplasia and skin flaking was unaffected in IMQ-treated Illr1 $1^{-/-}$mice, while neutrophil infiltration was inhibited. These 
A

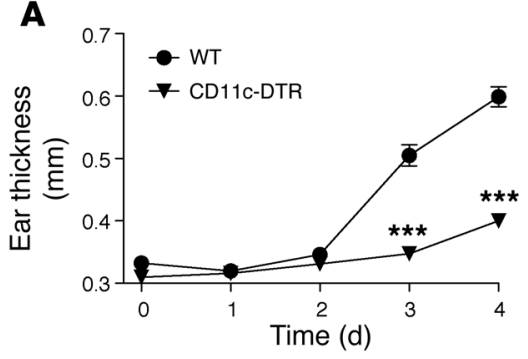

B

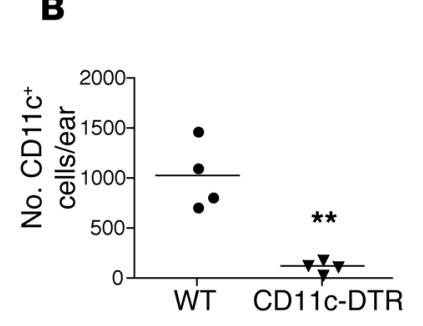

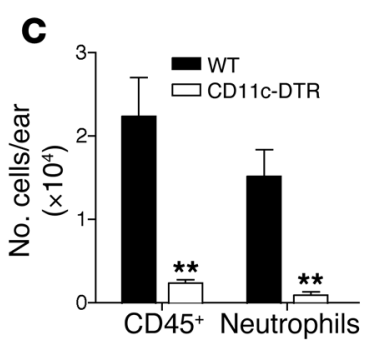

D

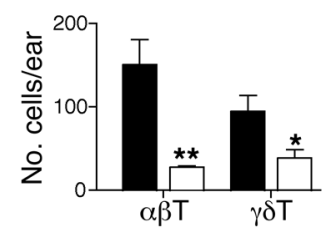

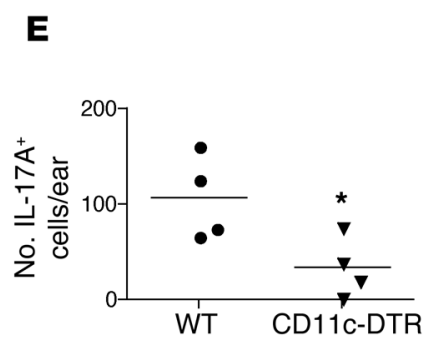
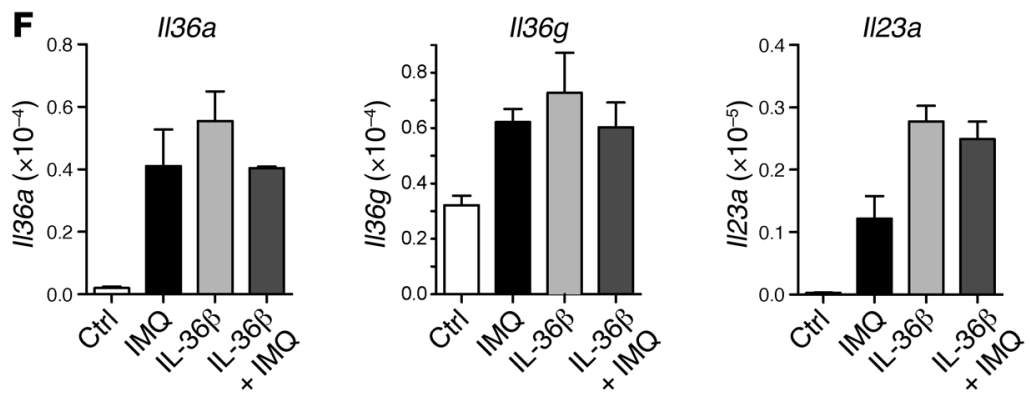

G

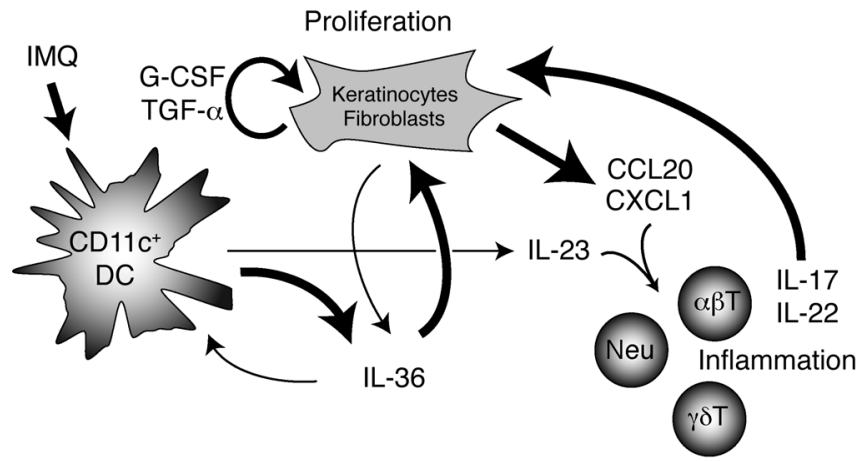

Figure 8

DCs produce IL-36 upon IMQ treatment and are pivotal for IMQ-induced cutaneous pathology. CD11c-DTR transgenic and WT control mice were injected with diphtheria toxin 6 hours prior to daily treatment with Aldara. (A) Ear thickness was monitored daily. Values show averages \pm SEM ( $n \geq 4)$. (B-E) On day 4, cells prepared from ears were analyzed by flow cytometry. Numbers of (B) CD11 $c^{+}$DCs, (C) CD $45^{+}$cells and neutrophils, (D) $\alpha \beta$ and $\gamma \delta$ T cells, and (E) IL-17A-producing cells per ear are shown. Symbols represent individual mice, and horizontal lines indicate averages of groups. (F) //36a, //36g, and I/23a mRNA expression in BMDCs cultured for 6 hours in the absence (Ctrl) or presence of IMQ, recombinant IL-36 $\beta$, or IMQ and IL-36 $\beta$, measured by real-time PCR. Values show mean \pm SEM normalized to HPRT. (G) Scheme illustrating development of psoriasiform dermatitis driven by DC-keratinocyte crosstalk via autocrine and paracrine IL-36 loops. Neu, neutrophil. ${ }^{*} P<0.05 ;{ }^{* \star} P<0.01 ;{ }^{* * *} P<0.001$.

findings uncouple neutrophil recruitment and tissue damage; the former being mediated by IL-1 and the latter by IL-36 in an IL-1independent manner.

Thus, comparing pathological manifestations in mice lacking IL-23, IL-17, IL-22, IL-36R, or IL-1R following IMQ treatment allowed us to rank the importance of these cytokines and their contribution for IMQ-induced psoriasiform dermatitis as follows: IL-36R $\geq$ IL-23 > IL-22, IL-17 > IL-1R.

So where and how do IL-36 cytokines act in the process of lesion development following IMQ encounter? Results from bone marrow chimeras revealed that IL-36R signaling on skin-resident cells, including keratinocytes and fibroblasts, but not on hematopoietic cells was essential for cutaneous pathology, indicating that IL-36 acted locally in the skin and was not essential for activation of innate or adaptive immune cells, including DCs and T cells. Yet DCs were essential to kick off the pathologic cytokine network, as they responded to IMQ with production of IL-36 and IL-23
(Figure 8F), while keratinocytes and dermal mesenchymal cells did not respond to IMQ due to the absence of TLR7 (Figure 7B and ref. 48). Stimulation of BMDCs with IL-36 agonists triggered IL-23 and IL-36 cytokine expression (Figure 8F), possibly together with other proinflammatory factors (37), comparably to TLR7 ligation by IMQ. Consequently, depletion of all CD $11 \mathrm{c}^{+}$cells, including conventional and plasmacytoid DCs as well as Langerhans cells, prevented disease induction. While depletion of langerin ${ }^{+}$DCs did not change IMQ-induced skin pathology, suggesting that Langerhans cells are not critically involved in this model (B.E. Clausen, personal communication), plasmacytoid DCs known to express high levels of TLR7 remain to be tested (49). Our data suggest that TLR7 engagement on conventional and/or plasmacytoid DCs triggers IL-36 production that acts in cis (autocrine) to promote the DC proinflammatory response and in trans (paracrine) to upregulate IL-36 cytokine production by skin-resident cells, including keratinocytes and fibroblasts. However, it is 
the IL-36R signaling on skin-resident cells that is crucial for development of psoriasiform lesions, as demonstrated by the results of the IL-36R/WT crisscross bone marrow chimeras. Indeed, culture of epidermal keratinocytes and dermal mesenchymal cells with IL-36 agonists potently induced IL-36 cytokine expression (Figure 7A) concomitant with the upregulation of chemokines that promote recruitment of neutrophils and dermal CCR $\sigma^{+} \gamma \delta \mathrm{T}$ cells (50). In addition, IL-36 triggered expression of keratinocyte mitogens by epidermal and dermal cells.

Our results suggest that autocrine IL-36 production by epidermal and dermal skin-resident cells may account for the remaining skin swelling that we have observed in the absence of IL-23, IL-17, and IL-22. However, dysregulated keratinocyte growth and differentiation, together with the production of antimicrobial peptides, is certainly amplified by IL-17A and IL-22 produced by the expanded dermal $\gamma \delta$ T cells (29). Although human keratinocytes were reported to produce IL-23 (51), IL-12p40 expression was undetectable in CD45- cells sorted from epidermis and dermis and stimulated with IMQ, IL-36 $\beta$, or IL-36 (data not shown), indicating that keratinocytes and dermal mesenchymal cells do not produce IL-23 under these conditions and that DCs are solely responsible for IL-23 production.

Taken together, we have established a central IL-36-mediated crosstalk between DCs and skin-tissue cells, such as keratinocytes and fibroblasts. Our results suggest the mechanism illustrated in Figure 8G. TLR7 engagement by IMQ triggers IL-23 and IL-36 cytokine production in DCs. While DC-derived IL-36 may augment the DC inflammatory response in cis (autocrine), it is mainly required for activation of keratinocytes and dermal mesenchymal cells in trans (paracrine). When activated, they produce IL-36 in an autocrine fashion along with chemokines that attract neutrophils (i.e., CXCL1) and dermal CCR $6^{+} \gamma \delta \mathrm{T}$ cells (i.e., CCL20) and growth factors that promote keratinocyte hyperproliferation, such as G-CSF and TGF- $\alpha$.

Due to increased understanding of the molecular mechanisms of immune pathology, current therapies use biologics targeting endogenous mediators of inflammation, such as TNF- $\alpha$, IL-23, or IL-17, that have shown efficacy in clinical trials $(1,2,52)$. However, inhibition of these cytokines, which are critical for certain types of immune responses, may render individuals susceptible to infection, underlining the need for tissue-specific targets. Our data, together with recent reports of patients with pustular psoriasis having mutations in the $I L 36 R N$ gene $(33,34)$, suggest that blocking IL-36R signaling might be a promising approach for the specific treatment of psoriasis.

\section{Methods}

Mice and antibodies. Il36r/- (31), Il36rn-/- (generated as described below), Illr1 $1^{-/-}$(53), Il17 $a^{-/-}$(54) (provided by Y. Iwakura, The Institute of Medical Science, University of Tokyo, Tokyo, Japan), Il23a-/- (55) (provided by R. Kastelein, Schering-Plough Biopharma, Palo Alto, California, USA), and $I l 22^{-/}$mice (56) were bred and maintained under specific pathogen-free conditions in our animal facility (BioSupport). All knockout mice were backcrossed to C57BL/6>7 generations. C57BL/6 mice were purchased from Charles River Inc. For experiments, age- and sex-matched mice (at 7 to 12 weeks of age) were used. The following antibodies from BioLegend were used: FITC-labeled anti-CD45.1 and anti-IL-17A; PerCP/Cy5.5-labeled anti-CD11b; PerCP-labeled streptavidin; PE/Cy7-labeled anti-CD45.2; and APC-labeled anti-CD11c, anti-CD45.1, and anti-IFN- $\gamma$. The following antibodies and reagents were purchased from eBioscience: FITC-labeled anti-
CD4 and anti-Ly-6G; PE-labeled anti-TCR $\beta$, anti-CD4, and anti-MHCII; APC-labeled anti-GR-1; biotin-labeled anti-TCR $\gamma \delta$; and the viability dye eFluor780. Recombinant IL-36 3 (aa 31-183) was purchased from R\&D Systems (catalog no. 7060-ML). R837 (IMQ) was purchased from Tocris.

Generation of knockout mice. Il36rn $\mathrm{rn}^{-/-}$mice were generated using a targeting vector designed to remove part of exon 1 and exon 2 by homologous recombination. 129S5/SvEv embryonic stem cells were transfected with the targeting vector by electroporation. Targeted embryonic stem clones were identified by Southern blot analysis and microinjected into C57BL/6 blastocysts. PCR was used for routine genotyping of the mice. The following primers were used: WT exon 1, 5'-TGGAGCTCATGATGGTTCTG-3'; WT intron 1, 5'-AGGATCCTGCTCAGTTCTTCC-3'; KO 5' arm, 5'-AAGGGCAGATCTGATGGATGC-3'; Neo, 5'-ATGACTGGGCACAACAGACA-3'. Il36rn-targeted mice were backcrossed at least 7 times on C57BL/6 background before performing the experiments.

Treatment of mice. For induction of psoriasiform dermatitis, mouse ears were treated as described previously (11) for 7 consecutive days with Aldara Crème containing $5 \%$ Imiquimodum (provided by MEDA Pharma GmbH). For neutralization of IL-22, mice were treated with $200 \mu \mathrm{g}$ anti-IL-22 (AM 22.1) or control anti-human IL-9R mAb (AH9R4) 4 hours prior application of Aldara at days 0,3 , and 5 .

Generation of bone marrow chimeras. Bone marrow cells were obtained from flushing femurs and tibias of donor WT CD45.1 and Il36r-1CD45.2 mice Lethally irradiated recipient mice (WT CD45.1 and Il36r-/CD45.2 mice) were then reconstituted by intravenous injection of bone marrow cells. Recipient mice were treated with antibiotics (Borgal 24\%, Veterinaria AG) for 5 weeks. Treatment with Aldara was performed 8 weeks after reconstitution.

Histopathology of ear skin. At day 7, mice were euthanized, and ears were removed and fixed in $95 \%$ ethanol/1\% aceton for subsequent embedding in paraffin. Tissue sections were deparaffinized and stained with H\&E for histological analysis.

Cell preparation. Single-cell suspensions from auricular lymph nodes were prepared by digestion with $1 \mathrm{mg} / \mathrm{ml}$ collagenase type IV (Sigma-Aldrich) and subsequent pressing of the organ through $40-\mu \mathrm{m}$ pore size strainers (BD Biosciences). To obtain a single-cell suspension from ears, ear halves were separated and cut into small pieces before collagenase digestion. Following digestion, the tissue was mechanically disrupted and pressed through $40-\mu \mathrm{m}$ pore size strainers. For preparation of epidermal and dermal skin-resident cells, ear halves were separated and incubated in $0.1 \%$ Trypsin IMDM for 50 minutes at $37^{\circ} \mathrm{C}$. Dermis and epidermis were separated, cut into small pieces, and digested in $0.1 \%$ Trypsin IMDM plus $0.1 \mathrm{mg} / \mathrm{ml}$ DNase for 30 minutes at $37^{\circ} \mathrm{C}$. Dermis was further digested with collagenase for 30 minutes at $37^{\circ} \mathrm{C}$. Single-cell suspension was made, and cells were washed once with IMDM 10\% FCS, stained, and sorted on the FACSAria (BD Biosciences). CD45- epidermal cells were plated out in defined keratinocyte medium (Gibco) (containing $10^{-10} \mathrm{M}$ cholera toxin), and CD45- dermal mesenchymal cells were plated out in pure IMDM at $10^{5}$ cells per well in a 96-well plate. After overnight starvation, cells were stimulated for 8 hours as indicated in Figure 7.

Immunofluorescence, surface staining, and analysis. Cells were initially stained with the viability dye eFLuor780 in PBS. Successively, cells were incubated with $\mathrm{Fc}$ receptor blocking $\mathrm{mAb}$ (clone $2.4 \mathrm{G} 2$ ) in PBS $2 \% \mathrm{FCS}$, after which staining with relevant fluorescently labeled antibodies was performed. For subsequent flow cytometric analysis using a FACSCanto II (BD Biosciences), cells were washed after a 15-minute incubation time and resuspended in PBS $2 \%$ FCS. FACS data was then analyzed using FlowJo (Tree Star Inc.).

In vitro restimulation and intracellular staining. To analyze cytokine production, single-cell suspensions were incubated for 2 hours at $37^{\circ} \mathrm{C}$ with PMA $\left(10^{-7} \mathrm{M}\right.$; Sigma-Aldrich), monensin ( $2 \mathrm{mg} / \mathrm{ml}$; Sigma-Aldrich), and ionomycin 
( $1 \mathrm{mg} / \mathrm{ml}$; Sigma-Aldrich). After performing surface stainings as described above, cells were fixed with $4 \%$ formalin and permeabilized with PBS $2 \%$ FCS supplemented with $0.5 \%$ saponin. Intracellular stainings with fluorescently labeled antibodies were performed for 30 minutes in PBS 2\% FCS with $0.2 \%$ saponin. For flow cytometric analysis, cells were washed and resuspended in PBS $2 \%$ FCS.

DC culture and stimulation. BMDCs were generated from bone marrow cells cultured for 10 days in complete RPMI medium supplemented with GM-CSF. At day 10, cells were stimulated with IMQ (R837) at $5 \mu \mathrm{g} / \mathrm{ml}$ and/or recombinant IL-36 $\beta$ (10 ng/ml). After 6 hours, supernatant was removed, and the cells were lysed in TRI Reagent and processed for RT-PCR as described below.

Quantitative RT-PCR. Total RNA was isolated from ears using TRI Reagent (Molecular Research Center Inc.) and reverse transcribed with GoScript (Promega). Quantitative real-time RT-PCR was performed with Brilliant SYBR Green (AGILENT-Stratagene) on an iCycler (Bio-Rad Laboratories). Expression was normalized to G6PD or HPRT as indicated in figure legends. The following primers were used: IL-36 $\alpha$ (forward) 5'-TGCCCACTCATTCTGACCCA-3' and (reverse) 5'-GTGCCACAGAGCAATGTGTC-3'; IL-36 $\gamma$ (forward) 5'-ATGGACACCCTACTTTGCTG-3' and (reverse) $5^{\prime}$-TGTCCGGGTGTGGTAAAACA-3'; IL-23p19 (forward) 5'-CACCTCCCTACTAGGACTCAGC-3' and (reverse) 5'-CTGCCACTGCTGACTAGAAC-3'; G-CSF (forward) 5'-TGCACTATGGTCAGGACGAG-3' and (reverse) 5 '-GGGGTGACACAGCTTGTAGG-3'; TGF- $\alpha$ (forward) $5^{\prime}$-CTGAGTGACTCACCCGTGGC-3' and (reverse) $5^{\prime}$-TCTGCATGCTCACAGCGAAC-3'; TLR7 (forward) 5'-CCACCAGACCTCTTGATTCC-3' and (reverse) 5'-CCATCGAAACCCAAAGACTC-3'; CXCL-1 (forward) 5'-GCCTATCGCCAATGAGCTG-3' and (reverse) 5'-ATTCTTGAGTGTGGCTATGA-3'; CCL20 (forward) 5'-AATCTGTGTGCGCTGATCCA-3' and (reverse) 5'-CCTTGGGCTGTGTCCAATTC-3'; GAPDH (forward) 5'-AAATTCAACGGCACAGTCAAG-3' and (reverse) 5'-TGGTGGTGAAGACACCAGTAG-3'; and G6PD (forward) 5'-CTACAGGTTCAGATGATGTC-3' and (reverse) 5'-CAGCTTCTCCTTCTCCATTG- ${ }^{\prime}$.

Statistics. Two-tailed paired and unpaired $t$ tests were performed using Prism 4.0 (GraphPad software). A $P$ value of less than 0.05 was considered significant. Data presented show values \pm SEM, if not indicated otherwise in figure legends.

Study approval. All animal experiments were approved by the veterinary authorities of the Canton of Zurich (Kantonales Veterinäramt Zürich).

\section{Acknowledgments}

We thank Sarah-Jane Smyth for generous support, John Sims for critical comments on the manuscript, and M. Kisielow and A. Schütz (ETH Flow Core Facility) for cell sorting. M. Kopf acknowledges funding of the project by the Swiss National Science Foundation (SNF grants 3100A0_100233 and 310030_124922).

Received for publication February 21, 2012, and accepted in revised form August 30, 2012.

Address correspondence to: Manfred Kopf or Jan Kisielow, Institute of Molecular Health Sciences, ETH Zürich, Schafmattstrasse 22, 8093 Zürich, Switzerland. Phone: 41.44.6336475; Fax: 41.44.6331350; E-mail: Manfred.Kopf@ethz.ch (M. Kopf), Jan. Kisielow@env.ethz.ch (J. Kisielow).

Brian Abel's present address is: Singapore Immunology Network, Agency for Science, Technology and Research, Singapore.
1. Lowes MA, Bowcock AM, Krueger JG. Pathogenesis and therapy of psoriasis. Nature. 2007; 445(7130):866-873.

2. Nestle FO, Kaplan DH, Barker J. Psoriasis. NEngl J Med. 2009;361(5):496-509.

3. Nestle FO, Di Meglio P, Qin JZ, Nickoloff BJ. Skin immune sentinels in health and disease. Nat Rev Immunol. 2009;9(10):679-691.

4. Boyman O, Hefti HP, Conrad C, Nickoloff BJ, Suter $\mathrm{M}$, Nestle FO. Spontaneous development of psoriasis in a new animal model shows an essential role for resident $\mathrm{T}$ cells and tumor necrosis factoralpha. J Exp Med. 2004;199(5):731-736.

5. Villadsen LS, et al. Resolution of psoriasis upon blockade of IL-15 biological activity in a xenograft mouse model. J Clin Invest. 2003;112(10):1571-1580.

6. Ma HL, et al. IL-22 is required for Th17 cell-mediated pathology in a mouse model of psoriasis-like skin inflammation. J Clin Invest. 2008;118(2):597-607.

7. Kopp T, Lenz P, Bello-Fernandez C, Kastelein RA, Kupper TS, Stingl G. IL-23 production by cosecretion of endogenous p19 and transgenic p40 in keratin 14/p40 transgenic mice: evidence for enhanced cutaneous immunity. J Immunol. 2003; 170(11):5438-5444.

8. Groves RW, Mizutani H, Kieffer JD, Kupper TS. Inflammatory skin disease in transgenic mice that express high levels of interleukin 1 alpha in basal epidermis. Proc Natl Acad Sci U S A. 1995; 92(25):11874-11878.

9. Li AG, Wang D, Feng XH, Wang XJ. Latent TGFbeta 1 overexpression in keratinocytes results in a severe psoriasis-like skin disorder. EMBO J. 2004; 23(8):1770-1781.

10. Chan JR, et al. IL-23 stimulates epidermal hyperplasia via TNF and IL-20R2-dependent mechanisms with implications for psoriasis pathogenesis. J Exp Med. 2006;203(12):2577-2587.

11. van der Fits $L$, et al. Imiquimod-induced psoriasis- like skin inflammation in mice is mediated via the IL-23/IL-17 axis. J Immunol. 2009;182(9):5836-5845.

12. Rajan N, Langtry JA. Generalized exacerbation of psoriasis associated with imiquimod cream treatment of superficial basal cell carcinomas. Clin Exp Dermatol. 2006;31(1):140-141.

13. Wu JK, Siller G, Strutton G. Psoriasis induced by topical imiquimod. Australas J Dermatol. 2004; 45(1):47-50.

14. Nakajima K, et al. Distinct roles of IL-23 and IL-17 in the development of psoriasis-like lesions in a mouse model. J Immunol. 2011;186(7):4481-4489.

15. Tonel G, et al. Cutting edge: A critical functional role for IL-23 in psoriasis. J Immunol. 2010; 185(10):5688-5691

16. Zheng $\mathrm{Y}$, et al. Interleukin-22, a $\mathrm{T}(\mathrm{H}) 17$ cytokine, mediates IL-23-induced dermal inflammation and acanthosis. Nature. 2007;445(7128):648-651.

17. Kisielow J, Kopf M, Karjalainen K. SCART scavenger receptors identify a novel subset of adult gammadelta T cells. J Immunol. 2008;181(3):1710-1716.

18. Martin B, Hirota K, Cua DJ, Stockinger B, Veldhoen M. Interleukin-17-producing gammadelta $T$ cells selectively expand in response to pathogen products and environmental signals. Immunity. 2009; 31(2):321-330.

19. Rachitskaya AV, et al. Cutting edge: NKT cells constitutively express IL-23 receptor and RORgammat and rapidly produce IL-17 upon receptor ligation in an IL-6-independent fashion. J Immunol. 2008; 180(8):5167-5171.

20. Chung Y, et al. Critical regulation of early Th17 cell differentiation by interleukin-1 signaling. Immunity. 2009;30(4):576-587.

21. Doisne JM, et al. Cutting edge: crucial role of IL-1 and IL-23 in the innate IL-17 response of peripheral lymph node NK1.1- invariant NKT cells to bacteria. J Immunol. 2011;186(2):662-666.

22. Sutton CE, Lalor SJ, Sweeney CM, Brereton CF,
Lavelle EC, Mills KH. Interleukin-1 and IL-23 induce innate IL-17 production from gammadelta T cells, amplifying Th17 responses and autoimmunity. Immunity. 2009;31(2):331-341.

23. Cargill $\mathrm{M}$, et al. A large-scale genetic association study confirms IL12B and leads to the identification of IL23R as psoriasis-risk genes. Am J Hum Genet. 2007;80(2):273-290.

24. Krueger GG, et al. A human interleukin-12/23 monoclonal antibody for the treatment of psoriasis. NEngl J Med. 2007;356(6):580-592.

25. Leonardi CL, et al. Efficacy and safety of ustekinumab, a human interleukin-12/23 monoclonal antibody, in patients with psoriasis: 76-week results from a randomised, double-blind, placebo-controlled trial (PHOENIX 1). Lancet. 2008; 371(9625):1665-1674.

26. Nair RP, et al. Genome-wide scan reveals association of psoriasis with IL-23 and NF-kappaB pathways. Nat Genet. 2009;41(2):199-204.

27. Sims JE, Smith DE. The IL-1 family: regulators of immunity. Nat Rev Immunol. 2010;10(2):89-102.

28. Debets R, et al. Two novel IL-1 family members, IL-1 delta and IL-1 epsilon, function as an antagonist and agonist of NF-kappa B activation through the orphan IL-1 receptor-related protein 2.J Immunol. 2001;167(3):1440-1446.

29. Carrier $Y$, et al. Inter-regulation of Th17 cytokines and the IL-36 cytokines in vitro and in vivo: implications in psoriasis pathogenesis. J Invest Dermatol. 2011;131(12):2428-2437.

30. Johnston A, et al. IL-1F5, -F6, -F8, and -F9: a novel IL-1 family signaling system that is active in psoriasis and promotes keratinocyte antimicrobial peptide expression. J Immunol. 2011;186(4):2613-2622.

31. Blumberg $\mathrm{H}$, et al. Opposing activities of two novel members of the IL-1 ligand family regulate skin inflammation. J Exp Med. 2007;204(11):2603-2614.

32. Blumberg H, et al. IL-1RL2 and its ligands contribute 
to the cytokine network in psoriasis. Jimmunol. 2010; 185(7):4354-4362.

33. Onoufriadis A, et al. Mutations in IL36RN/IL1F5 are associated with the severe episodic inflammatory skin disease known as generalized pustular psoriasis. Am J Hum Genet. 2011;89(3):432-437.

34. Marrakchi S, et al. Interleukin-36-receptor antagonist deficiency and generalized pustular psoriasis. NEngl J Med. 2011;365(7):620-628.

35. Muhr P, Zeitvogel J, Heitland I, Werfel T, Wittmann M. Expression of interleukin (IL)-1 family members upon stimulation with IL-17 differs in keratinocytes derived from patients with psoriasis and healthy donors. BrJ Dermatol. 2011;165(1):189-193.

36. Towne JE, Garka KE, Renshaw BR, Virca GD, Sims JE. Interleukin (IL)-1F6, IL-1F8, and IL-1F9 signal through IL-1Rrp2 and IL-1RAcP to activate the pathway leading to NF-kappaB and MAPKs.J Biol Chem. 2004;279(14):13677-13688.

37. Vigne S, et al. IL-36R ligands are potent regulators of dendritic and T cells. Blood. 2011;118(22):5813-5823.

38. Cai Y, et al. Pivotal role of dermal IL-17-producing gammadelta $T$ cells in skin inflammation. Immunity. 2011;35(4):596-610.

39. Pantelyushin S, et al. Rorgammat+ innate lymphocytes and gammadelta $\mathrm{T}$ cells initiate psoriasiform plaque formation in mice. J Clin Invest. 2012; 122(6):2252-2256

40. Towne JE, et al. Interleukin-36 (IL-36) ligands require processing for full agonist (IL-36alpha, IL-36beta, and IL-36gamma) or antagonist (IL-36Ra) activity. J Biol Chem. 2011;286(49):42594-42602.

41. Gray EE, Suzuki K, Cyster JG. Cutting edge: Identification of a motile IL-17-producing gammadelta $\mathrm{T}$ cell population in the dermis. J Immunol. 2011; 186(11):6091-6095

42. Liang SC, et al. Interleukin (IL)-22 and IL-17 are coexpressed by Th17 cells and cooperatively enhance expression of antimicrobial peptides. J Exp Med. 2006;203(10):2271-2279.

43. Van Belle AB, et al. IL-22 is required for imiquimod-induced psoriasiform skin inflammation in mice. J Immunol. 2012;188(1):462-469.

44. Szabowski A, et al. c-Jun and JunB antagonistically control cytokine-regulated mesenchymal-epidermal interaction in skin. Cell. 2000; 103(5):745-755

45. Aksentijevich I, et al. An autoinflammatory disease with deficiency of the interleukin-1-receptor antagonist. N Engl J Med. 2009;360(23):2426-2437.

46. Reddy S, et al. An autoinflammatory disease due to homozygous deletion of the IL1RN locus. $N$ Engl J Med. 2009;360(23):2438-2444.

47. Shepherd J, Little MC, Nicklin MJ. Psoriasis-like cutaneous inflammation in mice lacking interleukin-1 receptor antagonist. J Invest Dermatol. 2004; 122(3):665-669.

48. Lebre MC, et al. Human keratinocytes express functional Toll-like receptor 3, 4, 5, and 9. J Invest Dermatol. 2007;127(2):331-341.
49. Drobits B, et al. Imiquimod clears tumors in mice independent of adaptive immunity by converting pDCs into tumor-killing effector cells. J Clin Invest. 2012;122(2):575-585.

50. Li Z, Burns AR, Miller SB, Smith CW. CCL20, gammadelta $\mathrm{T}$ cells, and IL-22 in corneal epithelial healing. FASEB J. 2011;25(8):2659-2668.

51. Piskin G, Sylva-Steenland RM, Bos JD, Teunissen MB. In vitro and in situ expression of IL-23 by keratinocytes in healthy skin and psoriasis lesions: enhanced expression in psoriatic skin. J Immunol. 2006; 176(3):1908-1915.

52. Kopf M, Bachmann MF, Marsland BJ. Averting inflammation by targeting the cytokine environment. Nat Rev Drug Discov. 2010;9(9):703-718.

53. Labow $M$, et al. Absence of IL-1 signaling and reduced inflammatory response in IL-1 type I receptor-deficient mice. J Immunol. 1997;159(5):2452-2461.

54. Nakae S, et al. Antigen-specific T cell sensitization is impaired in IL-17-deficient mice, causing suppression of allergic cellular and humoral responses. Immunity. 2002;17(3):375-387.

55. Cua DJ, et al. Interleukin-23 rather than interleukin-12 is the critical cytokine for autoimmune inflammation of the brain. Nature. 2003; 421(6924):744-748

56. Kreymborg K, et al. IL-22 is expressed by Th17 cells in an IL-23-dependent fashion, but not required for the development of autoimmune encephalomyelitis. J Immunol. 2007;179(12):8098-8104 